\title{
The Maxwell field on the Schwarzschild spacetime: behaviour near spatial infinity
}

\author{
Juan Antonio Valiente Kroon * \\ School of Mathematical Sciences, Queen Mary, University of London, \\ Mile End Road, London E1 4NS, United Kingdom.
}

September 24, 2018

\begin{abstract}
The behaviour of the Maxwell field near one of the spatial infinities of the Schwarzschild solution is analysed by means of the transport equations implied by the Maxwell equations on the cylinder at spatial infinity. Initial data for the Maxwell equations will be assumed to be expandable in terms of powers of a coordinate $\rho$ measuring the geodesic distance to spatial infinity (in the conformal picture) and such that the highest possible spherical harmonics at order $p$ are $2^{p}$-polar ones. It is shown that if the $2^{p}$-polar harmonics at order $p$ in the initial data satisfy a certain regularity condition then the solutions to the transport equations at orders $p$ and $p+1$ are completely regular at the critical sets where null infinity touches spatial infinity. On the other hand, the solutions to the transport equations of order $p+2$ contain, in general, logarithmic singularities at the critical sets. It is expected that the ideas and techniques developed in the analysis of this problem can be employed to discuss the more challenging case of the transport equations implied by the conformal Einstein equations, and thus provide a way of constructing a proof of a certain rigidity conjecture concerning the developments of conformally flat initial data sets.
\end{abstract}

Keywords: General Relativity, asymptotic structure, Maxwell equations, spatial infinity.

\section{Introduction}

In [13] the following rigidity conjecture concerning the development of conformally flat initial data for the Einstein vacuum equations sets was put forward:

Conjecture. If an initial data set for the Einstein vacuum equations which is time symmetric and conformally flat in a neighbourhood $\mathcal{B}_{a}(i)$ of infinity yields a development with a smooth null infinity, then the initial data set is Schwarzschildean in $\mathcal{B}_{a}(i)$.

There is further evidence for generalisations of this conjecture to more general classes of initial data - see e.g. 12, 14, 15. A presumptive proof of such a conjecture would entail two aspects:

(I) A deep understanding of the mechanisms of the asymptotic expansions that are obtained using Friedrich's construction of the cylinder at spatial infinity - see e.g. [3, 5].

(II) The construction of estimates which allow to get around the manifest degeneracy of the Einstein conformal equations at the critical sets were null infinity "touches" spatial infinity - see e.g. [4.

The present article explores a set of ideas on how to deal with the aspect (I) mentioned above.

Friedrich's seminal work on the $i^{0}$ problem has rendered a representation of spatial infinity which exploits to the maximum the conformal properties of the spacetime by choosing a convenient

*E-mail address: j.a.valiente-kroon@qmul.ac.uk 
gauge. In particular, spatial infinity is represented in this gauge by an extended set, the cylinder at spatial infinity, $\mathcal{I}$ - see 3 . This framework uses the extended conformal field equations - as given in, say, 2, 3] - to pose a regular initial value problem at spatial infinity.

The cylinder at spatial infinity can be regarded as a limit set of incoming and outgoing light cones. It has the remarkable property of being a total characteristic of the conformal Einstein propagation equations. This property implies that the propagation equations reduce in their entirety to an interior system of transport equations upon evaluation at $\mathcal{I}$. This allows to transport information from some initial data prescribed on some initial hypersurface $\mathcal{S}$ up to the critical sets $\mathcal{I}^{ \pm}$where spatial infinity touches null infinity. Let $\phi=\left(\phi_{0}, \phi_{1}, \phi_{2}, \phi_{3}, \phi_{4}\right)$ denote the components of the rescaled Weyl tensor in a particular gauge and with respect to a particular frame - to be described in the subsequent sections. Then the transport equations at $\mathcal{I}$ allow to calculate $\left.\partial_{\rho}^{p} \phi\right|_{\mathcal{I}}$ — where $\rho$ is some radial coordinate measuring the distance to $\mathcal{I}$ - on the whole of $\mathcal{I}$ — and in particular at $\mathcal{I}^{ \pm}$— if the value of $\partial_{\rho}^{p} \phi$ on $\mathcal{I}^{0}=\mathcal{I} \cap \mathcal{S}$ is given and the lower order derivatives $\left.\partial_{\rho}^{k} \phi\right|_{\mathcal{I}}, k=0, \ldots, p-1$ are known. This unfolding of the evolution process can be thought of as the construction of the Taylor like expansions of the form

$$
\phi \sim \sum_{p} \frac{1}{p !}\left(\left.\partial_{\rho}^{p} \phi\right|_{\mathcal{I}}\right) \rho^{p} .
$$

A general observation is that the derivatives $\left.\partial_{\rho}^{p} \phi\right|_{\mathcal{I}}$ contain logarithmic divergences at $\mathcal{I}^{ \pm}$. By logarithmic divergences at, say $\tau= \pm 1$, it will be understood the appearance expressions of the form

$$
(1-\tau)^{k_{-}} \ln (1-\tau), \quad(1+\tau)^{k_{+}} \ln (1+\tau) .
$$

Note how the exponents $k_{-}$and $k_{+}$regulate the smoothness of the term. The logarithmic divergences appearing in the solutions of the transport equations implied by the conformal Einstein equations can be grouped in two types:

(i) those appearing from general linear aspects of the equations;

(ii) and those arising from the particular nonlinear aspects of the equations.

The logarithmic divergences of (i) are well understood and their preclusion imposes mild conditions on the initial data - see [3, 11. Not surprisingly, it is the understanding of the logarithmic divergences of type (ii) which poses the real challenge. The discovery of a particular class of this logarithms led to conjecture 1. At the moment, there are no general results regarding the behaviour of this kind of logarithms. The only knowledge available is what has been inferred from the calculation of the expansions (1) up to a finite order - say $p=7,8$ - using computer algebra methods.

With regards to the coefficients $\left(\left.\partial_{\rho}^{p} \phi\right|_{\mathcal{I}}\right)$ in the expansions (1), one would like to prove a result on the lines of:

Subconjecture. Given a time symmetric initial data which is conformally flat on $\mathcal{B}_{a}(i)$, and if $\left.\partial_{\rho}^{p} \phi\right|_{\mathcal{I}}$ are smooth at $\mathcal{I}^{ \pm}$for all $p$, then $\left.\partial_{\rho} \phi\right|_{\mathcal{I}^{0}}$ is Schwazschildean.

In other words, if one starts with a general time symmetric, conformally flat initial data set and one works out the behaviour of the derivatives $\left.\partial_{\rho}^{p} \phi\right|_{\mathcal{I}}$ at $\mathcal{I}^{ \pm}$, then one will observe that generically there are logarithmic terms. If one removes the parts of the initial data giving rise to these divergences at every order $p$, then one will observe that the jet $\left\{\left.\partial_{\rho} \phi\right|_{\mathcal{I}^{0}}, p=0,1, \ldots\right\}$ is equal to the one obtained from looking at the $t=$ constant slice of the Schwarzschild solution in isotropic coordinates. In order to make these ideas work, one needs the understanding of the asymptotic expansions which was alluded to in point (I).

The complexity and vast number of equations which has to be controlled when dealing with the conformal Einstein equations makes developing the required understanding a daunting endeavour. That is the reason why it is desirable to have simpler "toy models" on which diverse techniques could be tested and from which underlying structures could be apprehended. Devising such a toy model is a delicate issue. Of course, one wishes for a tractable model, but at the same time one would like to avoid simplifying things too much and render the analysis trivial. 
The obvious "toy model" in the form of a spin-2 zero-rest-mass field propagating close to one of the spatial infinities of the Schwarzschild spacetime is not an adequate one in view of the existence of an obstruction in the form of the Buchdahl constraint which precludes the consideration of generic fields in the analysis - see e.g. [1. This problem is overdetermined.

Due to the above considerations, attention is turned to another possible toy model: the propagation of the Maxwell field (spin-1 zero-rest-mass field) in the vicinity of one of the spatial infinities of the Schwarzschild spacetime. A direct application of the ideas of, say, [11 shows the existence of logarithmic divergences at the sets $\mathcal{I}^{ \pm}$of the underlying Schwarzschild spacetime which are due to generic linear features of the Maxwell equations which are shared with the conformal Einstein equations - the type (i) divergences. An understanding of the mechanism behind these divergences, as it will be seen, poses no challenge. The real difficulties arise from the existence of another type of logarithmic divergences which are generated by the interaction of the Maxwell field with the background spacetime. A preliminary analysis of the analogue of the expansions (11) for this toy model using the computer algebra methods of [13] reveals that the inner workings of the logarithmic divergences are similar to those of the nonlinear propagation of the gravitational field.

The main goal of the present work is to develop a procedure of tracking down which parts of the initial data are responsible for specific logarithmic divergences at the critical sets. To this end it shall be assumed that one has a class of initial data for the Maxwell equations expandable in powers of a geodesic distance $\rho$ to infinity. Further, it will be assumed that the coefficients of this expansion at say, order $\rho^{p}$ admit at most $2^{p}$-polar harmonics - this happens if, for example, the initial data is analytic in a neighbourhood of infinity. Consistent with the discussion of the previous paragraphs, it will also be assumed that the initial data satisfies the regularity conditions precluding the appearance of logarithms of the so-called type (i). Given this class of initial data, the question is the following: at which order - if at all- do the $2^{p}$-polar terms in the initial data produce divergences at the critical sets? Transport equations for $2^{p}$-polar quantities appear for the first time at order $p$. The present analysis shows that the solutions at orders $p$ and $p+1$ do not contain logarithmic divergences - for order $p$ this is direct, but for order $p+1$ there is an intriguing "conspiracy" of terms taking place. Moreover, for order $p+2$ the solutions contain, in general, the logarithmic terms. A similar pattern - although with a further layer of complication - arises for the conformal Einstein field equations. Hence the interest of the present analysis.

It should be mentioned that there has recently been some related work on the peeling properties of solutions of the wave equation on a Schwarzschild background - see [7, 8]. These analyses can, most certainly, be extended to the case of the Maxwell equations. In any case, the scope, aims, techniques and motivations for these results are different to the ones in this article.

The article is structured as follows: section 2 briefly discusses briefly the Schwarzschild spacetime in the so-called F-gauge in which the construction of the cylinder at spatial infinity is carried out. Section 3 is concerned with the Maxwell equations in the present context. Section 4 is considers further properties of the transport equations implied by the Maxwell equations at the cylinder at spatial infinity, including some regularity conditions. Sections 5, 6 and 7 discuss, respectively, the solutions to the order $p, p+1$ and $p+2$ transport equations. There are some concluding remarks in section 8 . Finally, there are three appendices including the definitions of some spinorial objects, the first orders of the asymptotic expansions of the Schwarzschild solution, and some properties of Jacobi polynomials which are used in the analysis.

\section{The Schwarzschild spacetime in the F-gauge}

The Schwarzschild spacetime was discussed in reference [3] as an example of the implementation of the framework of the cylinder at spatial infinity —or F-gauge. This representation will be used to analyse the propagation of the Maxwell field near spatial infinity. Some of the relevant features of this construction are reviewed.

The Schwarzschild metric in isotropic coordinates is given by

$$
\tilde{g}=\left(\frac{1-m / 2 r}{1+m / 2 r}\right)^{2} \mathrm{~d} t^{2}+\left(1+\frac{m}{2 r}\right)^{4}\left(\mathrm{~d} r^{2}+r^{2} \mathrm{~d} \sigma^{2}\right) .
$$


Now, consider the time symmetric hypersurface $\tilde{\mathcal{S}}$. Writing $\tilde{h}=\Omega^{-2} h$, one can introduce a conformal metric and conformal factor given by

$$
h=-\left(\mathrm{d} \rho^{2}+\rho^{2} \mathrm{~d} \sigma^{2}\right), \quad \Omega=\frac{\rho^{2}}{(1+\rho m / 2)^{2}},
$$

where the radial coordinate $\rho=1 / r$ has been introduced. Let $i_{1}$ and $i_{2}$ denote the infinities corresponding to the two asymptotic ends of the hypersurface $\tilde{\mathcal{S}}$. Further, let $\mathcal{S}=\tilde{\mathcal{S}} \cup\left\{i_{1}, i_{2}\right\}$. Let $i=i_{1}$ denote the infinity corresponding to the locus $\rho=0$. The discussion in this article will be concerned with the domain of influence, $J^{+}\left(\mathcal{B}_{a}(i)\right)$ of a sufficiently small ball, $\mathcal{B}_{a}(i)$, of radius $a$ based on $i$. The point $i$ can be blown up to a 2 -sphere, $\mathcal{S}^{2}$. With this idea in mind introduce the set $\mathcal{C}_{a}=\left(\mathcal{B}_{a}(i) \backslash i\right) \cup \mathcal{S}^{2}$.

The use of a gauge based on conformal Gaussian coordinates to discuss the structure of $\mathcal{B}_{a}(i)$ leads to a conformal factor for the portion of spacetime under consideration which is given by

$$
\Theta=\frac{\Omega}{\kappa}\left(1-\tau^{2} \frac{\kappa^{2}}{\omega^{2}}\right)
$$

where

$$
\omega=\frac{2 \Omega}{\sqrt{\left|D^{i} \Omega D_{i} \Omega\right|}}=\rho(1+\rho m / 2)
$$

and $\kappa>0$ is a smooth function such that $\kappa=\tilde{\kappa} \rho$ with $\tilde{\kappa}(i)=1$. The function $\kappa$ encodes the remaining conformal freedom in the setting. In what follows, and in order to ease the calculations let

$$
\kappa=\rho .
$$

The coordinate $\tau$ is an affine parameter of conformal geodesics whose tangent at $\tau=0$ is parallel to the normal of $\mathcal{S}$. The coordinate $\rho$ can be extended off $\mathcal{S}$ by requiring it to be constant along the aforementioned conformal geodesics. "Angular coordinates" can be extended in a similar fashion.

In view of the conformal factor (5) define the manifold

$$
\mathcal{M}_{a, \kappa}=\left\{(\tau, q) \mid q \in \mathcal{C}_{a},-\frac{\omega}{\kappa} \leq \tau \leq \frac{\omega}{\kappa}\right\},
$$

such that $J^{+}\left(\mathcal{B}_{a}(i)\right) \subset \mathcal{M}_{a, \kappa}$, and the following relevant subsets thereof:

$$
\begin{aligned}
& \mathcal{I}=\left\{(\tau, q) \in \mathcal{M}_{a, \kappa}|\rho(q)=0,| \tau \mid<\frac{\omega}{\kappa}\right\}, \\
& \mathcal{I}^{ \pm}=\left\{(\tau, q) \in \mathcal{M}_{a, \kappa} \mid \rho(q)=0, \tau= \pm 1\right\}, \\
& \mathcal{I}^{0}=\left\{(\tau, q) \in \mathcal{M}_{a, \kappa} \mid \rho=0, \tau=0\right\} \\
& \mathscr{I}^{ \pm}=\left\{(\tau, q) \in \mathcal{M}_{a, \kappa} \mid q \in \mathcal{B}_{a}(i) \backslash \mathcal{I}^{0}, \tau= \pm \frac{\omega}{\kappa}\right\},
\end{aligned}
$$

denoting, respectively, the cylinder at spatial infinity, the critical sets where spatial infinity touches null infinity, the intersection of the cylinder at spatial infinity with the initial hypersurface $\mathcal{S}$, and the two components of null infinity.

It will be convenient to work with a space-spinor formalism - see $[9]$. In order to write down the field equations, introduce a null frame $c_{A A^{\prime}}$ satisfying $g\left(c_{A A^{\prime}}, c_{B B^{\prime}}\right)=\epsilon_{A B} \epsilon_{A^{\prime} B^{\prime}}$. Let $\tau_{A A^{\prime}}$ -with normalisation $\tau^{A A^{\prime}} \tau_{A A^{\prime}}=2$ - be tangent to the conformal geodesics of which $\tau$ is a parameter. The frame can be split in the form

$$
c_{A A^{\prime}}=\frac{1}{2} \tau_{A A^{\prime}} \tau^{C C^{\prime}} c_{C C^{\prime}}-\tau^{B}{ }_{A^{\prime}} c_{A B},
$$

with

$$
\tau^{A A^{\prime}} c_{A A^{\prime}}=\sqrt{2} \partial_{\tau}, \quad c_{A B}=\tau_{(A}{ }^{B^{\prime}} c_{B) B^{\prime}} .
$$

For the purpose of the present investigation, the following choice is made:

$$
c_{00^{\prime}}=\frac{1}{\sqrt{2}}\left(\left(1+c^{0}\right) \partial_{\tau}+c^{1} \partial_{\rho}\right), \quad c_{11^{\prime}}=\frac{1}{\sqrt{2}}\left(\left(1-c^{0}\right) \partial_{\tau}-c^{1} \partial_{\rho}\right) .
$$


The remaining vectors of the frame, $c_{01^{\prime}}$ and $c_{10^{\prime}}$ must then be tangent to the spheres $\{\tau=$ constant, $\rho=$ constant $\}$, and thus cannot define smooth vector fields everywhere. To avoid this difficulty all possible tangent vectors $c_{01^{\prime}}$ and $c_{10^{\prime}}$ will be considered. This results in a 5dimensional submanifold of the bundle of normalised spin frames. Rotations $c_{01^{\prime}} \rightarrow \mathrm{e}^{\mathrm{i} \alpha}, \alpha \in \mathbb{R}$ leave this submanifold invariant. Hence it defines a subbundle with structure group $U(1)$ which projects into $\mathcal{M}_{a, \kappa}$. All the relevant structures will be lifted to the subbundle, which in an abuse of notation will be again denoted by $\mathcal{M}_{a, \kappa}$.

The introduction of the bundle space $\mathcal{M}_{a, \kappa}$ and of the frame $c_{A A^{\prime}}$ in our formalism implies that all relevant quantities have a definite spin weight and hence admit a characteristic - spherical harmonics - expansion in terms of some functions $T_{j}{ }_{l}^{k}$ associated with unitary representations of $S U(2, \mathbb{C})$ — see e.g. [3, [5] for a more detailed discussion in this respect. Further, one can introduce differential operators $X, X_{+}$and $X_{-}$defined by their action on the functions $T_{j}{ }_{l}^{k}$. It is noted that with the help of these operators one can write

$$
c_{A A^{\prime}}=c_{A A^{\prime}}^{\mu} \partial_{\mu}=c_{A A^{\prime}}^{0} \partial_{\tau}+c_{A A^{\prime}}^{1} \partial_{\rho}+c_{A A^{\prime}}^{+} X_{+}+c_{A A^{\prime}}^{-} X_{-} .
$$

In addition to the frame $c_{A A^{\prime}}$, the geometry of $\mathcal{M}_{a, \kappa}$ is described by means of the associated connection $\Gamma_{A A^{\prime} B C}$, the spinorial counterpart of the Ricci tensor of a Weyl connection $\Theta_{A A^{\prime} B B^{\prime}}$ and the rescaled Weyl spinor $\phi_{A B C D}$. The unprimed (space-spinor) version of the connection spinor is given by $\Gamma_{A B C D}=\tau_{B}{ }^{B^{\prime}} \Gamma_{A B^{\prime} C D}, \Gamma_{A A^{\prime} C D}=\Gamma_{A B C D} \tau^{B}{ }_{A^{\prime}}$, which is decomposed as

$$
\Gamma_{A B C D}=\frac{1}{\sqrt{2}}\left(\xi_{A B C D}-\chi_{(A B) C D}\right)-\frac{1}{2} \epsilon_{A B} f_{C D} .
$$

Similarly, one considers $\Theta_{A B C D}=\tau_{C}{ }^{A^{\prime}} \tau_{D}{ }^{B^{\prime}} \Theta_{A A^{\prime} B B^{\prime}}$. The explicit spherical symmetry of the spacetime justifies the following Ansatz in terms of irreducible spinors:

$$
\begin{aligned}
& c_{A B}^{0}=c^{0} x_{A B}, \quad c_{A B}^{1}=c^{1} x_{A B}, \quad c_{A B}^{-}=c^{-} y_{A B}, \quad c_{A B}^{+}=c^{+} z_{A B}, \\
& f_{A B}=f x_{A B}, \quad \xi_{A B C D}=\xi\left(\epsilon_{A C} x_{B D}+\epsilon_{B D} x_{A C}\right), \\
& \chi_{(A B) C D}=\chi_{2} \epsilon_{A B C D}^{2}+\chi_{h} h_{A B C D}, \\
& \Theta_{A B C D}=\Theta_{2} \epsilon_{A B C D}^{2}+\Theta_{h} h_{A B C D}+\Theta_{x} \epsilon_{A B} x_{C D}, \quad \phi_{A B C D}=\phi \epsilon_{A B C D}^{2} .
\end{aligned}
$$

The definitions of the irreducible spinors introduced above are given in the appendix. The manifest spherical symmetry of this representation implies that the functions $c^{0}, c^{1}, c^{ \pm}, f, \xi, \chi_{2}, \chi_{h}$, $\phi$ have spin-weight 0 . Furthermore they only contain the function $T_{0}{ }_{0}^{0}=1$.

The functions $c^{0}, c^{1}, c^{ \pm}, f, \xi, \chi_{2}, \chi_{h}, \phi$ are determined by solving the conformal propagation equations discussed in 3 with the appropriate initial data. The problem of reconstructing the conformal Schwarzschild solution from the given data amounts to finding a solution $u=u(\tau, \rho ; m)$ of an initial value problem of the type

$$
\partial_{\rho} u=F(u, \tau, \rho ; m), \quad u(0, \rho ; m)=u_{0}(\rho ; m),
$$

with analytic functions $F$ and $u_{0}$. The solution with $m=0$ corresponds to a portion of the conformal Minkowski spacetime, in which the only non-vanishing components of the solution are given by

$$
c^{0}=-\tau, \quad c^{1}=\rho, \quad c^{ \pm}=1, \quad f=1 .
$$

Since in this case the solution exists for all $\tau, \rho \in \mathbb{R}$, it follows from standard results of ordinary differential equations that for a given $m$ there is sufficiently small $\rho_{0}$ such that there is an analytic solution to the system (16) which extends beyond $\mathscr{I}$ for $|\rho|<\left|\rho_{0}\right|$. Hence, one can recover the portion of the Schwarzschild spacetime which lies near null and spatial infinity, $J^{+}\left(\mathcal{B}_{a}(i)\right)$, if $a$ is taken to be small enough.

It follows from the above discussion that the coefficients that are obtained from solving the transport propagation equations on the cylinder at spatial infinity — as discussed in [3, 13] correspond to the first terms in the expansions of the solutions of (16). See the appendix for a list of the relevant expansions. 


\section{The Maxwell field}

As it is common when working with spinors, consider the Maxwell tensor written as

$$
F_{A A^{\prime} B B^{\prime}}=\phi_{A B} \epsilon_{A^{\prime} B^{\prime}}+\bar{\phi}_{A^{\prime} B^{\prime}} \epsilon_{A B}
$$

Then the Maxwell equations are equivalent to

$$
\nabla^{A A^{\prime}} \phi_{A B}=0
$$

that is, the spin-1 zero-rest-mass field equations. Recall, as well, that if the conformal weight of $\phi_{A B}$ is chosen properly, the vacuum Maxwell equations are conformally invariant. The spinor $\phi_{A B}$ is symmetric and thus, one can write

$$
\phi_{A B}=\phi_{0} \epsilon_{A B}^{0}+\phi_{1} \epsilon_{A B}^{1}+\phi_{2} \epsilon_{A B}^{2} .
$$

In the F-gauge, and using a space-spinor decomposition, the above equation is equivalent to the following propagation equations:

$$
\begin{gathered}
\left(\sqrt{2}-2 c_{01}^{0}\right) \partial_{\tau} \phi_{0}+2 c_{00}^{0} \partial_{\tau} \phi_{1}-2 c_{01}^{\alpha} \partial_{\alpha} \phi_{0}+2 c_{00}^{\alpha} \partial_{\alpha} \phi_{1} \\
=\left(2 \Gamma_{0011}-4 \Gamma_{1010}\right) \phi_{0}+4 \Gamma_{1000} \phi_{1}-2 \Gamma_{0000} \phi_{2} \\
\sqrt{2} \partial_{\tau} \phi_{1}-c_{11}^{0} \partial_{\tau} \phi_{0}+c_{00}^{0} \partial_{\tau} \phi_{2}-c_{11}^{\alpha} \partial_{\alpha} \phi_{0}+c_{00}^{\alpha} \partial_{\alpha} \phi_{2} \\
=-\left(2 \Gamma_{1110}+f_{11}\right) \phi_{0}+2\left(\Gamma_{1100}+\Gamma_{0011}\right) \phi_{1}-\left(2 \Gamma_{0001}-f_{00}\right) \phi_{2} \\
\left(\sqrt{2}+2 c_{01}^{0}\right) \partial_{\tau} \phi_{2}-2 c_{11}^{0} \partial_{\tau} \phi_{1}+2 c_{01}^{\alpha} \partial_{\alpha} \phi_{2}-2 c_{11}^{\alpha} \partial_{\alpha} \phi_{1} \\
=-2 \Gamma_{1111} \phi_{0}+4 \Gamma_{0111} \phi_{1}+\left(2 \Gamma_{1100}-4 \Gamma_{0101}\right) \phi_{2} .
\end{gathered}
$$

In addition, one has the following constraint equation

$$
\begin{gathered}
c_{11}^{0} \partial_{\tau} \phi_{0}-2 c_{01}^{0} \partial_{\tau} \phi_{1}+c_{00}^{0} \partial_{\tau} \phi_{2}+c_{11}^{\alpha} \partial_{\alpha} \phi_{0}-2 c_{01}^{\alpha} \partial_{\alpha} \phi_{1}+c_{00}^{\alpha} \partial_{\alpha} \phi_{2} \\
=\left(2 \Gamma_{1110}-2 \Gamma_{(01) 11}\right) \phi_{0}+2\left(\Gamma_{0011}-\Gamma_{1100}\right) \phi_{1}+\left(2 \Gamma_{(01) 00}-\Gamma_{0001}\right) \phi_{2}
\end{gathered}
$$

Because of the symmetries of the Schwarzschild spacetime, the connection coefficients $\Gamma_{0011}, \Gamma_{1010}$, $\Gamma_{1100}$ and $\Gamma_{0101}$ are the only non-vanishing ones. The leading terms of frame and connection components are listed in the appendix B.

Writing $\phi=\left(\phi_{0}, \phi_{1}, \phi_{2}\right)$, the Maxwell propagation equations can be concisely expressed in the form

$$
\sqrt{2} E \partial_{\tau}+A^{A B} c_{A B}^{\mu} \partial_{\mu} \phi=B\left(\Gamma_{A B C D}\right) \phi
$$

where $E$ denotes the $(3 \times 3)$-unit matrix, $A^{A B} c_{A B}^{\mu}$ are $(3 \times 3)$-matrices depending on the coordinates and $B(\Gamma)$ is a matrix valued function of the connection $\Gamma_{A B C D}$. For consistency the constraint equation (22) will be written as

$$
F^{A B} c_{A B}^{\mu} \partial_{\mu} \phi=H\left(\Gamma_{A B C D}\right) \phi
$$

where $F^{A B} c_{A B}^{\mu}$ denotes $(1 \times 3)$ matrices, and $H$ is a function of the connection.

The system (23) shares the property with the Bianchi propagation system for the rescaled Weyl tensor and the propagation system for linearised gravity that its principal degenerates at critical sets $\mathcal{I}^{ \pm}$. More precisely, one has that

$$
\left.\left(\sqrt{2} E+A^{A B} c_{A B}^{0}\right)\right|_{\mathcal{I}}=\sqrt{2} \operatorname{diag}(1-\tau, 1,1+\tau) .
$$

Most non-smooth and/or polyhomogeneous behaviour of the solutions of the Maxwell equations at the conformal boundary can be traced back to precisely this degeneracy.

Another deciding property of the Maxwell equations in the aforediscussed form stems from the fact that $\left.A^{A B} c_{A B}^{1}\right|_{\mathcal{I}}=0$. From here it follows - see e.g. the discussion in [11] - that the equations imply an intrinsic system of transport equations on $\mathcal{I}$ which determines the solution on $\mathcal{I}$ in terms of the data implied by $\phi$ on $\mathcal{I}^{0}$. In other words, $\mathcal{I}$ is a total characteristic of (23) and (24). 
As in the case of the conformal Einstein field equations of [3], given an asymptotically flat spacetime one can produce, for the Maxwell field, a hierarchy of transport equations at the cylinder at spatial infinity out of the equations (23) and (24). Differentiating the latter equations with respect to $\rho$, say, $p$ times and evaluating them at the cylinder at spatial infinity $\mathcal{I}$ one obtains, by virtue of $\left.c_{A B}^{1}\right|_{\mathcal{I}}=0$ the equations

$$
\begin{aligned}
(\sqrt{2} E & \left.+A^{A B}\left(c_{A B}^{0}\right)^{(0)}\right) \partial_{\tau} \phi^{(p)}+A^{A B}\left(c_{A B}^{C}\right)^{(0)} \partial_{C} \phi^{(p)} \\
& =B\left(\Gamma_{A B C D}^{(0)}\right) \phi^{(p)}+\sum_{j=1}^{p}\left(\begin{array}{c}
p \\
j
\end{array}\right)\left(B\left(\Gamma_{A B C D}\right) \phi^{(p-j)}-A^{A B}\left(c_{A B}^{\mu}\right)^{(j)} \partial_{\mu} \phi^{(p-j)}\right) \\
F^{A B}\left(c_{A B}^{0}\right)^{(0)} \partial_{\tau} \phi^{(p)}+F^{A B}\left(c_{A B}^{C}\right)^{(0)} \partial_{C} \phi^{(p)} & \\
& =H\left(\Gamma_{A B C D}^{(0)}\right) \phi^{(p)}+\sum_{j=1}^{p}\left(\begin{array}{c}
p \\
j
\end{array}\right)\left(H\left(\Gamma_{A B C D}\right) \phi^{(p-j)}-F^{A B}\left(c_{A B}^{\mu}\right)^{(j)} \partial_{\mu} \phi^{(p-j)}\right)
\end{aligned}
$$

with $C= \pm$. It is noted that the principal part of the above equations is universal, in the sense that it depends only on the zeroth-order solution of the background metric. The first term on the right-hand side is also universal, while the remaining non-homogeneous terms depend on lower order terms of the Maxwell field and higher order terms of the background spacetime. As in the case of the gravitational field, provided some suitable initial data one can solve the above equations to any desired order in a recursive way.

The coefficients $\phi_{0}, \phi_{1}$ and $\phi_{2}$ have, respectively, spin-weights 1, 0 and -1 . Consistently with their spin-weight, the following Ansatz will be made:

$$
\phi_{j}=\sum_{p=|1-j|}^{\infty} \sum_{q=|1-j|}^{p} \sum_{k=0}^{2 q} \frac{1}{p !} a_{j, p ; q, k}(\tau) T_{2 q}{ }^{k}{ }_{q-1+j} \rho^{p},
$$

with $j=0,1,2$, and $a_{j, p ; 2 q, k}: \mathbb{R} \longrightarrow \mathbb{C}$.

The above assumption will allow to solve the hierarchy of transport equations (26a) and (26b) by "decomposing in modes". It is important to note that because the background terms in the transport equations only contain the function $T_{0}{ }_{0}^{0}=1$, it will not be necessary to linearise products of the form $T_{j_{1}}{ }_{m_{1}} m_{1} \times T_{j_{2}}{ }_{m_{2}}^{l_{2}}$ when performing the mode decomposition.

\subsection{Initial data for the Maxwell equations}

The equation (22) evaluated on $\tau=0$ reduces, for the Schwarzschild spacetime, to

$$
\rho \partial_{\rho} \phi_{1}+\frac{1}{2} X_{-} \phi_{0}-\frac{1}{2} X_{+} \phi_{2}=0 .
$$

The latter equation can be read as an equation (ODE) for $\phi_{1}$, if $\phi_{0}$ and $\phi_{2}$ are given on the initial hypersurface. In the present work, initial data for the Maxwell equations on the initial hypersurface $\mathcal{S}$ which when lifted to $\mathcal{C}_{a}$ is expandable in powers of $\rho$ will be considered. Accordingly, prescribe on $\mathcal{C}_{a}$ components $\phi_{0}$ and $\phi_{2}$ of the form

$$
\phi_{0}=\sum_{p=0}^{\infty} \sum_{q=1}^{p} \sum_{k=0}^{2 q} \frac{1}{p !} a_{0, p ; 2 q, k}(0) T_{q}{ }^{k}{ }_{q-1} \rho^{p}, \quad \phi_{2}=\sum_{p=0}^{\infty} \sum_{q=1}^{p} \sum_{k=0}^{2 q} \frac{1}{p !} a_{2, p ; q, k}(0) T_{2 q}{ }^{k}{ }_{q+1} \rho^{p},
$$

which correspond to the expansions of analytic $\phi_{0}$ and $\phi_{2}$ - see [3. One can use equation (22) to obtain the coefficients $a_{1, p ; 2 q, k}(0)$ in

$$
\phi_{1}=\sum_{p=0}^{\infty} \sum_{q=0}^{p} \sum_{k=0}^{2 q} \frac{1}{p !} a_{1, p ; q, k}(0) T_{2 q}{ }^{k}{ }_{q} \rho^{p},
$$

in terms of $a_{0, p ; 2 q, k}(0)$ and $a_{2, p ; 2 q, k}(0)$. It is noted that in this construction the coefficient $a_{1,0 ; 0,0}(0)$ - the electric charge - is not determined and can be prescribed freely. On the other hand, from equation (22) it follows that $a_{1, p ; 0,0}(0)=0$ for $p \geq 1$. 


\section{Further properties of the transport equations}

The discussion in this section is inspired from the treatment given in [3] of the transport equations for the Conformal Einstein equations. Besides being given as a reference, it will help to highlight the difficulties to be confronted when analysing the solutions of the equations (26a) and (26b) for an arbitrary order $p$.

\subsection{Reduction to an overdetermined system of ODE's}

The propagation transport equations implied by the Maxwell equations on the cylinder at spatial infinity of the Schwarzschild background are of the form:

$$
\begin{aligned}
& (1+\tau) \partial_{\tau} \phi_{0}^{(p)}+X_{+} \phi_{1}^{(p)}-(p-1) \phi_{0}^{(p)}=R_{0}^{(p)}, \\
& \partial_{\tau} \phi_{1}^{(p)}+\frac{1}{2}\left(X_{+} \phi_{2}^{(p)}+X_{-} \phi_{0}^{(p)}\right)=R_{1}^{(p)}, \\
& (1-\tau) \partial_{\tau} \phi_{2}^{(p)}+X_{-} \phi_{1}^{(p)}+(p-1) \phi_{2}^{(p)}=R_{2}^{(p)},
\end{aligned}
$$

while the constraint transport equation is given by

$$
\tau \partial_{\tau} \phi_{1}^{(p)}+\frac{1}{2} X_{+} \phi_{2}^{(p)}-\frac{1}{2} X_{-} \phi_{0}^{(p)}-p \phi_{1}^{(p)}=S^{(p)} .
$$

As mentioned before, the terms $R_{1}^{(p)}, R_{2}^{(p)}, R_{3}^{(p)}$ and $S^{(p)}$ depend solely on the lowers order solutions to the equations. Using the expansion Ansatz (27) it can be seen that the coefficients $a_{j, p ; q, k}$ satisfy the equations

$$
\begin{aligned}
& (1+\tau) a_{0, p ; q, k}^{\prime}+\sqrt{q(q+1)} a_{1, p ; q, k}-(p-1) a_{0, p ; q, k}=U_{0, p ; q, k}, \\
& a_{1, p ; q, k}^{\prime}+\frac{1}{2} \sqrt{q(q+1)}\left(a_{2, p ; q, k}-a_{0, p ; q, k}\right)=U_{1, p ; q, k}, \\
& (1-\tau) a_{2, p ; q, k}^{\prime}-\sqrt{q(q+1)} a_{1, p ; q, k}+(p-1) a_{2, p ; q, k}=U_{2, p ; q, k},
\end{aligned}
$$

and

$$
\tau a_{1, p ; q, k}^{\prime}+\frac{1}{2} \sqrt{q(q+1)}\left(a_{2, p ; q, k}+a_{0, p ; q, k}\right)-p a_{1, p ; q, k}=T_{p ; q, k},
$$

for $p \geq 1,1 \leq q \leq p$, and where again the nonhomogeneous terms $U_{0, p ; q, k}, U_{1, p ; q, k}, U_{2, p ; q, k}$ and $T_{p ; q, k}$ in the equations depend on the background spacetime and lower order $a_{j, p ; q, k}$ 's. The problem has been reduced to the analysis of an overdetermined system of ordinary differential equations. The last equation can be used to eliminate the unknown $a_{1, p ; q, k}$. In what remains of this section, attention will be focused on a fixed - but otherwise arbitrary - value of the indices $p, q, k$ in their range of definition which henceforth will often be suppressed in order to avoid cluttering the equations.

The coefficient $a_{1}$ can be eliminated from the following considerations by noting that

$$
-\frac{1}{2} \sqrt{q(q+1)}(1+\tau) a_{0}-\frac{1}{2} \sqrt{q(q+1)}(1-\tau) a_{2}+p a_{1}=\tau U_{1}-T
$$

from where one can solve for $a_{1}$. One is left with the system

$$
\begin{aligned}
& (1+\tau) a_{0}^{\prime}+\left(\frac{1}{2} q(q+1)(1+\tau)-p+1\right) a_{0}+\frac{1}{2 p} q(q+1)(1-\tau) a_{2} \\
& =U_{0}+\frac{1}{p} \sqrt{q(q+1)}\left(T-\tau U_{1}\right), \\
& (1-\tau) a_{2}^{\prime}-\frac{1}{2 p} q(q+1)(1+\tau) a_{0}+\left(-\frac{1}{2 p} q(q+1)(1-\tau)+p-1\right) a_{2} \\
& =U_{2}+\frac{1}{p} \sqrt{q(q+1)}\left(\tau U_{1}-T\right) .
\end{aligned}
$$


For future reference the above system will be written in matricial form as

$$
Y_{p ; q, k}^{\prime}(\tau)=A_{p ; q, k}(\tau) Y_{p ; q, k}(\tau)+B_{p ; q, k}(\tau),
$$

with $A_{p ; q, k}$ a $(2 \times 2)$-matrix, and $Y_{p ; q, k}, B_{p ; q, k}(2 \times 1)$-column vectors. Note that the Wronskian determinant $\operatorname{det} X_{p ; q}$ of the above system - satisfying $\left(\operatorname{det} X_{p ; q}\right)^{\prime}=\left(\operatorname{Tr} A_{p ; q, k}\right) X_{p ; q}$ - is given by

$$
\operatorname{det} X_{p ; q}=X_{p ; q}^{0}\left(1-\tau^{2}\right)^{p-1},
$$

where $X_{p ; q}^{0}$ is a constant. In order to calculate the fundamental matrix of the system consider the equations (again, the multisubindex begin supressed)

$$
\begin{aligned}
& \left(1-\tau^{2}\right) a_{0}^{\prime \prime}+(2-(2-2 p) \tau) a_{0}^{\prime}+(q+p)(q-p+1)=0, \\
& \left(1-\tau^{2}\right) a_{2}^{\prime \prime}+(-2-(2-2 p) \tau) a_{2}^{\prime}+(q+p)(q-p+1)=0,
\end{aligned}
$$

which are implied by the homogeneous parts of the equations (36a) and (36b). Equations (39a) and (39b) are so-called Jacobi equations

$$
D_{(n, \alpha, \beta)} a=(1-\tau) a^{\prime \prime}+(\beta-\alpha-(\alpha+\beta+2) \tau) a^{\prime}+n(n+\alpha+\beta+1) a=0,
$$

with

$$
\alpha=-1-p, \quad \beta=1-p, \quad n_{1}=p+q, \quad n_{2}=p-q-1 .
$$

Note that if $a_{0}(\tau)$ is a solution of equation (39a), then $a_{0}^{s}(\tau) \equiv a_{0}(-\tau)$ is a solution of equation (39b). A class of solutions is given by the Jacobi polynomials $P_{n}^{(\alpha, \beta)}(\tau)$ - see the appendix for their definition and relevant properties.

Using the definition of the polynomials, $P_{p+q}^{(-1-p,-p+1)}$ are seen to vanish identically. On the other hand,

$$
Q_{2}=P_{p+q}^{(-p-1,-p+1)}
$$

is a solution of degree $n_{2}=p-q-1$ for $p \geq 2,1 \leq q \leq p-1$. Using some identities of the polynomials one finds that

$$
Q_{1}=\left(\frac{1-\tau}{2}\right)^{p+1} P_{q-1}^{(p+1,-p+1)}
$$

is another solution, $p \geq 2,1 \leq q \leq p-1$. Clearly $Q_{1}$ and $Q_{2}$ are linearly independent as $n_{1}>n_{2}$. Another solution is given by

$$
Q_{3}=\left(\frac{1+\tau}{2}\right)^{p-1} P_{q+1}^{(-p-1, p-1)}
$$

for $p \geq 2,1 \leq q \leq p-1$. A direct calculation shows that the fundamental matrix, $X_{p, q}$ of the system is given by

$$
X_{p, q}=\left(\begin{array}{cc}
Q_{1} & (-1)^{q+1} Q_{3} \\
(-1)^{q+1} Q_{3}^{s} & Q_{1}^{s}
\end{array}\right)
$$

for $p \geq 2,1 \leq q \leq p-1$. The notation $Q_{1}^{s}(\tau) \equiv Q_{1}(-\tau), Q_{3}^{s}(\tau) \equiv Q_{3}(-\tau)$ has been used again. Note that the fundamental matrix does not depend on $k$. The inverse of $X_{p, q}$ is given by

$$
X_{p, q}^{-1}=\frac{1}{X_{p ; q}^{0}}\left(\begin{array}{cc}
\frac{(1+\tau)^{2}}{(1-\tau)^{p-1}} P_{q-1}^{(p+1,1-p)}(-\tau) & \frac{(-1)^{q}}{(1-\tau)^{p-1}} P_{q+1}^{(-p-1, p-1)}(\tau) \\
\frac{(-1)^{q}}{(1+\tau)^{p-1}} P_{q+1}^{(-p-1, p-1)}(-\tau) & \frac{(1-\tau)^{2}}{(1+\tau)^{p-1}} P_{q-1}^{(p+1,1-p)}(\tau)
\end{array}\right)
$$

The case $p=q, p \geq 1$ is solved separately. It is noted that

$$
D_{(2 p,-p-1,-p+1)}\left(\left(\frac{1-\tau}{2}\right)^{p+1}\left(\frac{1+\tau}{2}\right)^{p-1} a_{0}\right)=\left(\frac{1-\tau}{2}\right)^{p+1}\left(\frac{1+\tau}{2}\right)^{p-1} D_{(0, p+1, p-1)} a_{0}
$$


where

$$
D_{(0, p+1, p-1)} a_{0}=\left(1-\tau^{2}\right) a_{0}^{\prime \prime}+(-2-(2 p+2)) a_{0}^{\prime}
$$

so that

$$
a_{0}=\left(\frac{1-\tau}{2}\right)^{p+1}\left(\frac{1+\tau}{2}\right)^{p-1}\left(C_{1}+C_{2} \int_{0}^{\tau} \frac{\mathrm{d} s}{(1+s)^{p}(1-s)^{p+2}}\right),
$$

with $C_{1}$ and $C_{2}$ some integration constants. Noting, again, that $a_{2}(\tau)=a_{0}^{s}(\tau) \equiv a_{0}(-\tau)$ one obtains the following

Proposition 1. For $p=q$, the fundamental matrix $X_{p ; q}$ of the system (36a) and (36b) is polynomial if and only if

$$
a_{0, p ; q, k}(0)=a_{2, p ; q, k}(0) .
$$

The latter is the analogue, in our context, of the regularity conditions of [3] and [11.

In what follows, it will be assumed that the initial data for the propagation equations (21a)(21c) is constructed such that condition (50) holds.

\subsection{The solution to the nonhomogenous part}

Once the fundamental matrix of the system (36a) - (36b) is known, the solution is given by

$$
\left(\begin{array}{c}
a_{0, p ; q, k}(\tau) \\
a_{2, p ; q, k}(\tau)
\end{array}\right)=X_{p, q}(\tau)\left[X_{p, q}^{-1}(0)\left(\begin{array}{c}
a_{0, p ; q, k}(0) \\
a_{2, p ; q, k}(0)
\end{array}\right)+\int_{0}^{\tau} X_{p, q}^{-1}(s) B_{p, q}(s) \mathrm{d} s\right] .
$$

As seen before, the entries of $X_{p ; q}^{-1}$ are rational functions with - in the worst of cases - poles of order $p-1$ at $\tau= \pm 1$. One can make the following direct but crucial statement:

Proposition 2. If the functions $U_{0, p ; q, k}, U_{1, p ; q, k}, U_{2, p ; q, k}$ and $T_{p ; q, k}$ in equations (21a)-(21c) and (22) are polynomial in $\tau$ - that is, if the lower order functions $a_{j, p^{\prime} ; q^{\prime}, k^{\prime}}(\tau)$ with $0 \leq p^{\prime}<p$, $0 \geq q^{\prime} \geq p^{\prime}, 0 \geq k^{\prime} \geq 2 q^{\prime}$ are polynomials in $\tau$ - then the solutions given by (51) consist of polynomials in $\tau$ and of products of polynomials in $\tau$ times the functions $\ln (1-\tau)$ and $\ln (1+\tau)$.

Proof. The entries of the vector $X_{p, q}^{-1} B_{p, q}$ are, respectively, of the form

$$
\frac{f_{0}(\tau)}{(1+\tau)(1-\tau)^{p-1}} \quad \text { and } \quad \frac{f_{2}(\tau)}{(1-\tau)(1+\tau)^{p-1}},
$$

where $f_{0}(\tau)$ and $f_{2}(\tau)$ are polynomials in $\tau$. Decomposition in partial fractions of the first entry renders an expression containing $(1+\tau)^{-1},(1-\tau)^{-n}$ with $n=1, \ldots, p-1$, and a polynomial. Analogously for the second entry, where one finds a decomposition in terms of $(1-\tau)^{-1},(1+\tau)^{-n}$ with $n=1, \ldots, p-1$, and a polynomial. The integration produces, in the first entry, an expression containing $\ln (1 \pm \tau),(1-\tau)^{1-n}$ with $n=2, \ldots, p-1$ and a polynomial. Similarly for the second entry. Now, the multiplication by the fundamental matrix cancels out the rational expressions.

Remark 1. From the previous discussion it is clear that even if the regularity condition (50) holds, the solutions to the transport equations will contain $\ln (1 \pm \tau)$ terms unless some cancellations take place.

Remark 2. On the other hand, one could use an argument analogous to that of the above proof to show that if the lower order coefficients already contain $\ln (1 \pm \tau)$-singular terms - say because the regularity condition (50) is not satisfied, then the integration in equation (51) would render $\ln ^{2}(1 \pm \tau)$-terms. This type of analysis is, however, beyond the present discussion. 


\section{$5 \quad$ Expansions at order $p$}

From the hyperbolicity and linearity of the propagation equations, if for a given order $p$ a certain type of harmonics has not yet appeared in the initial data, it will also not be present in the solutions to the transport equations. For the class of initial data under consideration, terms containing harmonics of the form $T_{2 p}{ }^{k}{ }_{p-1+j}$ - which will be referred to as $2^{p}$-polar - appear firstly at order $p$. It follows that if $p=q$, then

$$
B_{p ; p, k}(\tau)=0,
$$

for the class of data under consideration - for all the lower terms $a_{j, p^{\prime} ; q^{\prime}, k^{\prime}}$ with $0 \leq p^{\prime} \leq p$, $|1-j| \leq q^{\prime} \leq p^{\prime}$ and $0 \leq k^{\prime} \leq 2 q^{\prime}$ can only contain at most $T_{2(p-1)}^{k} p-2+j$ functions. In the sequel, the following observation will be repeatedly used: the Schwarzschild solution contains only $T_{0}{ }_{0}$ sectors. Hence, all the products of coefficients of the Maxwell field and background terms are trivially expanded in terms of the functions $T_{2 q}{ }^{k} p-2+j$ present in the coefficients of the Maxwell field. This observation might seem trivial, but note that if one considers a representation of the Schwarzschild solution where the spherical symmetry is not manifest, a "mixing of modes" will occur - and in particular higher order harmonics would be generated, severely complicating the analysis. From here it follows that the first appearance in the solutions to the transport equations of terms containing $T_{2 p}{ }_{p-1+j}^{k}$ renders no logarithms if the regularity condition (50) is satisfied.

Proposition 3. If the regularity condition (50) holds, then the solutions $a_{0, p ; p, k}(\tau), a_{1, p ; p, k}(\tau)$ and $a_{2, p ; p, k}(\tau)$ of the transport equations (33a)-(33c) and (34) for $p=q$ and $k=0, \ldots, 2 p$ are polynomial in $\tau$. Moreover, one has that

$$
\begin{aligned}
& a_{0, p ; p, k}(\tau)=C_{p ; p, k}(1-\tau)^{p+1}(1+\tau)^{p-1}, \\
& a_{1, p ; p, k}(\tau)=C_{p ; p, k} \sqrt{\frac{p+1}{p}}(1-\tau)^{p}(1+\tau)^{p}, \\
& a_{2, p ; p, k}(\tau)=C_{p ; p, k}(1-\tau)^{p-1}(1+\tau)^{p+1},
\end{aligned}
$$

with $C_{p ; p, k}=a_{0, p ; p, k}(0)$.

\section{Expansions at order $p+1$}

If the condition (50) holds, then the first occasion where the harmonics $T_{2 p}{ }^{k} p-1+j$ can produce logarithms via the formula (51) is in the order $p+1$ of the transport equations. Quite remarkably, as it will be seen, this is not the case: cancellations occur which prevent the appearance of logarithms at this order. It is necessary to go up to order $p+2$ to find the first logarithms in the sectors $T_{2 p}{ }^{k} p-1+j$. More precisely, one has the following:

Proposition 4. If the regularity condition (50) is satisfied for $p \in \mathbb{N}, p \geq 1$ then the functions $a_{j, p ; 2 p, k}(\tau)$ are polynomial in $\tau$ - that is, they are logarithm free.

Remark. It must be emphasised that in view of the formula (51), the above result arises as the result of a remarkable collusion of terms which, in principle, there is no reason to expect. It would be very interesting to understand the deeper (possibly group theoretical) reasons behind this result.

Proof. The proof proceeds by an explicit calculation. The equations (36a) $-(36 \mathrm{~b})$ imply upon the replacement $p \rightarrow p+1, q \rightarrow p$ the system

$$
\begin{aligned}
& a_{0, p+1}^{\prime}-\frac{p}{2} \frac{(1-\tau)}{(1+\tau)} a_{0, p+1}+\frac{p}{2} \frac{(1-\tau)}{(1+\tau)} a_{2, p+1}=b_{0, p+1}, \\
& a_{2, p+1}^{\prime}+\frac{p}{2} \frac{(1+\tau)}{(1-\tau)} a_{2, p+1}-\frac{p}{2} \frac{(1+\tau)}{(1-\tau)} a_{0, p+1}=b_{2, p+1},
\end{aligned}
$$


where the subindex string $; p, k$ has been suppressed throughout for the ease of the presentation. In accordance with equation (26a) one has

$$
\begin{aligned}
& b_{0, p+1}=\frac{1}{1+\tau}\left(h_{0, p} a_{0, p}+h_{1, p} a_{1, p}+h_{2, p} a_{2, p}+\tilde{h}_{0, p} a_{0, p}^{\prime}+\tilde{h}_{1, p} a_{1, p}^{\prime}+\tilde{h}_{2, p} a_{2, p}^{\prime}\right), \\
& b_{2, p+1}=\frac{1}{1-\tau}\left(h_{2, p}^{s} a_{0, p}+h_{1, p}^{s} a_{1, p}+h_{0, p}^{s} a_{2, p}+\tilde{h}_{2, p}^{s} a_{0, p}^{\prime}+\tilde{h}_{1, p}^{s} a_{1, p}^{\prime}+\tilde{h}_{0, p}^{s} a_{2, p}^{\prime}\right),
\end{aligned}
$$

with $h_{j, p}$ and $\tilde{h}_{j, p}$ for $j=0,1,2$ some polynomials in $\tau$ arising from the order $0,1,2$ solutions of the transport equations for the Schwarzschild background - see appendix B. As before the notation $h_{j, p}^{s}(\tau) \equiv h_{j, p}(-\tau)$ has been used.

The order $p$ Maxwell transport equations can be used to eliminate $a_{0, p}^{\prime}, a_{1, p}^{\prime}$ and $a_{2, p}^{\prime}$ from the expressions for $b_{0, p+1}$ and $b_{2, p+1}$. Further, equation (35) with $q=p$ can be used to eliminate $a_{1, p+1}$. At the end one is left with

$$
\begin{aligned}
& b_{0, p+1}=\frac{1}{(1+\tau)^{2}}\left(f_{0} a_{0, p}+f_{2} a_{2, p}\right), \\
& b_{2, p+1}=\frac{1}{(1-\tau)^{2}}\left(f_{2}^{s} a_{0, p}+f_{0}^{s} a_{2, p}\right),
\end{aligned}
$$

with

$$
\begin{aligned}
& \begin{aligned}
f_{0}(\tau)=-\frac{1}{12} & m(p+1) \tau\left((-4 p-5) \tau^{6}+(-8 p-2) \tau^{5}+(25+16 p) \tau^{4}+(56 p+40) \tau^{3}\right. \\
& \left.+(30+48 p) \tau^{2}+24 \tau\right)
\end{aligned} \\
& \begin{aligned}
f_{2}(\tau)=\frac{1}{12} p(p+1) m\left((-4 p-5) \tau^{6}+(4 p+2) \tau^{5}+(29+28 p) \tau^{4}+(-16 p-8) \tau^{3}\right. \\
\left.\quad-(18 m+24 p) \tau^{2}\right)
\end{aligned}
\end{aligned}
$$

It follows then that

$$
X_{p+1, p}^{-1} B_{p, p+1}=\frac{C_{p ; p}}{X_{p+1, p}^{0}}\left(\begin{array}{c}
\frac{(1+\tau)^{p-1}}{(1-\tau)^{3}}\left(J_{1}^{s} F_{0}(1-\tau)^{2}+(-1)^{p} J_{3} F_{2}\right) \\
\frac{(1-\tau)^{p-1}}{(1+\tau)^{3}}\left(J_{1} F_{2}(1+\tau)^{2}+(-1)^{p} J_{3}^{s} F_{0}\right)
\end{array}\right),
$$

where the polynomials $J_{1}$ and $J_{3}$ arising from the fundamental matrix are given by

$$
\begin{aligned}
& J_{1}(\tau)=\frac{1}{2^{p+2}} P_{p-1}^{(p+2,-p)}(\tau), \\
& J_{3}(\tau)=\frac{1}{2^{p}} P_{p+1}^{(-p-2, p)}(\tau) .
\end{aligned}
$$

The polynomials $F_{0}$ and $F_{2}$ are given by

$$
\begin{aligned}
& F_{0}=(1-\tau)^{2} f_{0}+(1+\tau)^{2} f_{2}, \\
& F_{2}=(1-\tau)^{2} f_{2}^{s}+(1+\tau)^{2} f_{0}^{s},
\end{aligned}
$$

In order to determine whether the solutions of the transport equations at this order contain logarithms, one has to decide, say, whether

$$
H(\tau)=(1+\tau)^{p-1}\left((1-\tau)^{2} J_{1}^{s} F_{0}+(-1)^{p+1} J_{3} F_{2}\right)
$$

and its derivatives have zeros at $\tau=1$. Direct evaluation of formula (62) using the identities listed in the appendix for the values of the Jacobi polynomials at the terminal points $\tau= \pm 1$ render

$$
\begin{aligned}
& H(1)=2\left(1-p^{2}\right) m, \\
& H^{\prime}(1)=\frac{1}{3}(p+1)(5 m-7 p m), \\
& H^{\prime \prime}(1)=0 .
\end{aligned}
$$


Using the division algorithm one can write

$$
H(\tau)=(1-\tau)^{2} h_{2}(\tau)+(1-\tau) h_{1}(1)+H(1) .
$$

where $h_{1}(\tau)$ and $h_{2}(\tau)$ are some polynomials. Thus, $h_{2}(1)=0$, if $H^{\prime \prime}(1)=0$, whence

$$
H(\tau)=(1-\tau)^{3} h_{3}(\tau)+(1-\tau) h_{1}(1)+H(1) .
$$

Hence

$$
X_{p+1 ; p}^{-1} B_{p+1 ; p}=\frac{C_{p ; p}}{X_{p+1, p}^{0}}\left(\begin{array}{l}
h_{3}(\tau)+\frac{h_{1}(1)}{(1-\tau)^{2}}+\frac{H(1)}{(1-\tau)^{3}} \\
h_{3}^{s}(\tau)+\frac{h_{1}^{s}(1)}{(1+\tau)^{2}}+\frac{H^{s}(1)}{(1-\tau)^{3}}
\end{array}\right),
$$

from where it follows that the solutions $a_{0, p+1 ; p, k}(\tau), a_{1, p+1 ; p, k}(\tau), a_{2, p+1 ; p, k}(\tau)$ are polynomial as no $(1 \pm \tau)^{-1}$ terms have to be integrated. Note also, that the multiplication of $\int X_{p+1 ; p}^{-1} B_{p+1 ; p} \mathrm{~d} \tau$ by $X_{p+1 ; p}$ cancels out all rational expressions.

\section{$7 \quad$ The order $p+2$}

It is now shown how the $T_{2 p}{ }^{k}{ }_{q-1+j}$ harmonics at order $\mathcal{O}\left(\rho^{p}\right)$ in the initial data can give rise to solutions to the transport equations with logarithmic divergences at the critical sets $\mathcal{I}^{ \pm}$. The main result is the following:

Proposition 5. There are initial data satisfying the regularity condition (50) such that the functions $a_{0, p+2 ; p, k}(\tau), a_{1, p+2 ; p, k}(\tau)$ and $a_{2, p+2 ; p, k}(\tau)$ with $k=0, \ldots, 2 p$, solving equations (33a), (336) and (33C) contain logarithmic divergences at $\tau= \pm 1$.

Proof. A look at the integral formula (51) reveals that the order $p+1$ solutions $a_{0, p+1 ; p, k}(\tau)$, $a_{1, p+1 ; p, k}(\tau)$ and $a_{2, p+1 ; p, k}(\tau)$ are composed by two independent parts: one having to do with the homogeneous part of the solution which can be eliminated by setting $a_{0, p+1 ; p, k}(0)=a_{2, p+1 ; p, k}(0)$, $k=0, \ldots, 2 p$ in the initial data, and the other the nonhomogeneous solution proper which can be "turned off" by setting $a_{0, p ; p, k}(0)=0$-assuming the regularity condition (50) holds. For the purpose of exhibiting the existence of logarithmic divergences in the solutions to the order $p+2$ Maxwell transport equations it will suffice to analyse what happens when only the homogeneous terms of the order $p+1$ solutions are considered - exploiting the linearity of the transport equations. Let $Y_{p+1 ; p, k}^{h}$ denote these terms. From formula (51) one has that

$$
Y_{p+1}^{h}(\tau)=X_{p+1}(\tau) X_{p+1}^{-1}(0)\left(\begin{array}{c}
a_{0, p+1}(0) \\
a_{2, p+1}(0)
\end{array}\right),
$$

where again here and in the sequel the subindex string $; p, k$ has been suppressed. Consequently, performing the replacement $p \rightarrow p+2$ and $q \rightarrow p$ in the equations (36a - 36b and setting $a_{0, p}(\tau)=a_{1, p}(\tau)=a_{2, p}(\tau)=0$ - which is the case if $a_{0, p}(0)=0$ - one finds that the components $b_{0, p+2}$ and $b_{2, p+2}$ of the column vector $B_{p+2}$ containing the nonhomogeneous part of the system of ordinary differential equations to be solved are given by

$$
\begin{aligned}
& b_{0, p+2}=\frac{1}{(1+\tau)^{3}}\left(g_{0} a_{0, p+1}+g_{2} a_{2, p+1}\right), \\
& b_{2, p+2}=\frac{1}{(1-\tau)^{3}}\left(g_{0}^{s} a_{0, p+1}+g_{2}^{s} a_{2, p+1}\right),
\end{aligned}
$$

with the polynomials $g_{0}$ and $g_{2}$ given by

$$
\begin{aligned}
g_{0}(\tau)=\frac{m \tau}{12}(1+\tau)( & \left(4 p^{2}+9 p\right) \tau^{5}+\left(8 p^{2}+10 p-12\right) \tau^{4}-\left(16 p^{2}+33 p-4\right) \tau^{3} \\
& \left.-\left(56 p^{2}+112 p+8\right) \tau^{2}-\left(48 p^{2}+126 p+72\right) \tau-(24 p+48)\right),(690) \\
g_{2}(\tau)=-\frac{m \tau^{2}}{12}(1+\tau) & \left((4 p+9) \tau^{4}-(4 p+6) \tau^{3}\right. \\
& \left.-(28 p+57) \tau^{2}+(16 p+24) \tau+(24 p+42)\right)
\end{aligned}
$$


In the above expressions the order $p+1$ Maxwell transport equations have been used to eliminate the derivatives $a_{0, p+1}^{\prime}, a_{1, p+1}^{\prime}$ and $a_{2, p+1}^{\prime}$ that arise. Further, equation (35) has also been used to dispose of $a_{1, p+1}$.

For conciseness of the presentation, let

$$
X_{p+1}^{-1}(0) Y_{p+1}(0)=\left(\begin{array}{c}
z_{0} \\
z_{2}
\end{array}\right) \text {. }
$$

Hence, one has that

$$
Y_{p+1}^{h}=\left(\begin{array}{c}
a_{0, p+1}(\tau) \\
a_{2, p+1}(\tau)
\end{array}\right)=\left(\begin{array}{c}
z_{0}(1-\tau)^{p+2} J_{1}(\tau)+(-1)^{p+1} z_{2}(1+\tau)^{p} J_{3}(\tau) \\
z_{2}(1+\tau)^{p+2} J_{1}^{s}(\tau)+(-1)^{p} z_{0}(1-\tau)^{p} J_{3}^{s}(\tau)
\end{array}\right)
$$

where $J_{1}$ and $J_{3}$ are the polynomials (60a) - $60 \mathrm{~b}$ ) arising from the order $p+1$ fundamental matrix.

As in the proof of proposition 4 the crucial point in the argumentation is to analyse the decomposition in partial fractions of the entries of

$$
X_{p+2, p}^{-1} B_{p+2}=\frac{1}{X_{p+2, p}^{0}}\left(\begin{array}{l}
\frac{1}{(1-\tau)^{p+1}}\left(s_{0} a_{0, p+1}+s_{2} a_{2, p+1}\right) K_{1}^{s}+\frac{(-1)^{p}}{(1-\tau)^{p+3}}\left(s_{0}^{s} a_{0, p+1}+s_{2}^{s} a_{2, p+1}\right) K_{3} \\
\frac{1}{(1+\tau)^{p+1}}\left(s_{0}^{s} a_{0, p+1}+s_{2}^{s} a_{2, p+1}\right) K_{1}+\frac{(-1)^{p}}{(1+\tau)^{p+3}}\left(s_{0} a_{0, p+1}+s_{2} a_{2, p+1}\right) K_{3}^{s}
\end{array}\right),
$$

where it has been used that $g_{0}=(1+\tau) s_{0}$ and $g_{2}=(1+\tau) s_{2}$, and

$$
\begin{aligned}
& K_{1}(\tau)=\frac{1}{2^{p+3}} P_{p-1}^{(p+3,-p-1)}(\tau), \\
& K_{3}(\tau)=\frac{1}{2^{p+1}}(1+\tau)^{p+1} P_{p+1}^{(-p-3, p+1)}(\tau),
\end{aligned}
$$

are the Jacobi polynomials arising in the fundamental matrix $X_{p+2, p}$.

Contrary to what happens in the analysis of the order $p+1$, the functions $a_{0, p+1}$ and $a_{2, p+1}$ are not homogeneous in $(1 \pm \tau)^{m}$ for some integer $m$. This fact complicates the analysis of the decomposition in partial fractions of the expression (72). However, if one is interested in exhibiting the generic existence of logarithms in the solutions, their appearance for a restricted type of initial data suffices. With this idea in mind, set $z_{2}=0$ in equation (171) so that

$$
a_{0, p+1}(\tau)=z_{0}(1-\tau)^{p+2} J_{1}(\tau), \quad a_{2, p+1}(\tau)=z_{0}(-1)^{p}(1-\tau)^{p} J_{3}^{s}(\tau) .
$$

So, one finds that in particular

$$
\begin{gathered}
\frac{1}{(1-\tau)^{p+1}}\left(s_{0} a_{0, p+1}+s_{2} a_{2, p+1}\right) K_{1}^{s}+\frac{(-1)^{p}}{(1-\tau)^{p+3}}\left(s_{0}^{s} a_{0, p+1}+s_{2}^{s} a_{2, p+1}\right) K_{3} \\
=z_{0}(1-\tau) K_{1}^{s} J_{1} s_{0}+\frac{G(\tau)}{(1-\tau)^{3}}
\end{gathered}
$$

where

$$
G=(-1)^{p}(1-\tau)^{2} K_{1}^{s} J_{3}^{s} s_{2}+(-1)^{p}(1-\tau)^{2} K_{3} J_{1} s_{0}^{s}+K_{3} J_{3} s_{2}^{s} .
$$

As in the previous section, the problem reduces to calculating $G^{\prime \prime}$ and finding out whether it has zeros at $\tau=1$.

A direct calculation shows that

$$
\begin{aligned}
& G(1)=p(p+2)^{2} 2^{-2 p-1} m\left(\begin{array}{c}
2 p+1 \\
p+1
\end{array}\right), \\
& G^{\prime}(1)=-\frac{m}{12}(p+2)\left(3 p^{3}-4 p^{2}-16 p+2\right)\left(\begin{array}{c}
2 p+1 \\
p+1
\end{array}\right), \\
& G^{\prime \prime}(1)=\frac{m}{12}(p+2)\left(3 p^{4}-4 p^{3}-3 p^{2}-36 p-98\right)\left(\begin{array}{c}
2 p+1 \\
p+1
\end{array}\right) .
\end{aligned}
$$


It can be seen that the only real positive root of the polynomial in $\mathrm{p}, 3 p^{4}-4 p^{3}-3 p^{2}-36 p-98$, lies in the interval $(3,4)$ so that one can conclude that $G^{\prime \prime}(1) \neq 0$, for $p \geq 1$. Using arguments similar to those of the proof of proposition 4 one concludes that

$$
G(\tau)=(1-\tau)^{3} \tilde{h}_{3}(\tau)+(1-\tau)^{2} \tilde{h}_{2}(1)+(1-\tau) \tilde{h}_{1}(1)+G(1),
$$

for some polynomials $\tilde{h}_{1}, \tilde{h}_{2}$ and $\tilde{h}_{3}$, with $h_{2}(1) \neq 0$. Whence

$$
\frac{G(\tau)}{(1-\tau)^{3}}=\tilde{h}_{3}(\tau)+\frac{\tilde{h}_{2}(1)}{1-\tau}+\frac{\tilde{h}_{1}(1)}{(1-\tau)^{2}}+\frac{G(1)}{(1-\tau)^{3}} .
$$

Thus, it follows that the integration in formula (51) renders logarithmics terms $\ln (1 \pm \tau)$. Note, again, that the multiplication of the integral by $X_{p+2, p}$ cancels out all fractional terms in $(1 \pm \tau)$ so that the solution lies in the ring generated by polynomials in $\tau$ and $\ln (1 \pm \tau)$.

\section{Conclusions}

The present work has been a first attempt to understand, in a general way, the mechanisms logarithmic divergences arising at the critical sets $\mathcal{I}^{ \pm}$in the solutions to the transport equations at $\mathcal{I}$ implied by a Maxwell field propagating on a Schwarzschild background. The ultimate goal behind this programme is to develop ideas and methods which could be employed in the analysis of the much more challenging case of the behaviour of the transport equations implied by the Conformal Einstein equations. Preliminary calculations show that by the methods employed in this article it should be possible to at least obtain an analogue of propositions 3 and 4 for this case. That is, starting from initial data which are exactly Schwarzschildean up to order $p-1$, and with a first deviation from Schwarzschild data occurring at order $p$, it should be possible to fully integrate the order $p$ transport equations - note that in this case the system consists of 50 equations! - to show that if a certain regularity condition in the data holds - the analogous of condition (50) - then there are no logarithms in the solutions of the transport equations at order $p$ and $p+1$. Quite remarkably, some explicit calculations for concrete values of $p$ show that there should not be any logarithms in the solution at order $p+2$, and that the analogue of proposition 5 occurs in this case at order $p+3$. It is quite possible, however, that a proof of these last two observations is beyond the possibilities of the present methods, and that further ideas and insights have to be developed.

In the light of the evidence gathered in this article one can not avoid to feel that there is some powerful group theoretical structure lurking behind which is the responsible of the remarkable patterns that have been observed in both the case analysed in this article and more generally in the conformal Einstein field equations.

It would be of particular interest to reformulate the results obtained here in a context or language more in tone with ideas and concepts of Differential Galois theory, which in turn would render further insights - see e.g. 6] for a brief introduction to the ideas and goals of Differential Galois theory. For example, propositions 3 4 and 5 state that the Galois groups of the order $p$ and $p+1$ transport equations are trivial, while that of the order $p+2$ equations are not. Now, the question is the following: is it possible to obtain these results by - say- looking directly at the field equations and without having to solve almost explicitly the equations?

\section{Acknowledgements}

This research is funded by an EPSRC Advanced Research Fellowship. I thank the Max Planck Institute for Gravitational Physics (Albert Einstein Institute) for its hospitality during the course of a visit. I thank CM Losert V-K for a careful reading of the manuscript. 


\section{A Some spinors}

Let $\left\{o_{A}, \iota_{A}\right\}$ denote normalised spinor dyads, $\epsilon_{A B} O^{A} \iota^{B}=1$, where $\epsilon_{A B}$ is the standard alternating spinor. In the text, the following spinors have been used:

$$
\begin{aligned}
& \tau_{A A^{\prime}}=o_{A} o_{A^{\prime}}+\iota_{A} \iota_{A^{\prime}}, \\
& \epsilon_{A B}^{0}=o_{A} o_{B}, \quad \epsilon_{A B}^{1}=o_{\left(A \iota_{B}\right)}, \quad \epsilon_{A B}^{2}=\iota_{A} \iota_{B}, \\
& x_{A B}=\frac{1}{\sqrt{2}}\left(o_{A} \iota_{B}+\iota_{A} o_{B}\right), \quad y_{A B}=-\frac{1}{\sqrt{2}} \iota_{A} \iota_{B}, \quad z_{A B}=\frac{1}{\sqrt{2}} o_{A} o_{B}, \\
& \left.\epsilon_{A B C D}^{0}=o_{A} o_{B} o_{C} o_{D}, \quad \epsilon_{A B C D}^{1}=o_{(A} o_{B} o_{C} \iota_{D}\right), \cdots, \quad \cdots, \quad \epsilon_{A B C D}^{4}=\iota_{A} \iota_{B} \iota_{C} \iota_{D}, \\
& h_{A B C D}=-\epsilon_{A(C} \epsilon_{D) B} .
\end{aligned}
$$

\section{B The expansions of the Schwarzschild spacetime}

The following expansions of the field quantities on the F-gauge for the conformal Schwarzschild solution have been used in the discussion of the article. The expansions have been calculated using the computer algebra methods developed in [13.

$$
\begin{aligned}
& c^{0}=-\tau+\left(\frac{4}{3} m \tau^{3}-\frac{1}{3} m \tau^{5}\right) \rho+\frac{1}{2 !}\left(\frac{1}{7} m^{2} \tau^{9}+\frac{8}{7} m^{2} \tau^{7}-3 m^{2} \tau^{5}-2 m^{2} \tau^{3}\right) \rho^{2}+\mathcal{O}\left(\rho^{3}\right), \\
& c^{1}=\rho+\frac{1}{2 !}\left(-4 m \tau^{2}+\frac{2}{3} \tau^{4}\right) \rho^{2}+\frac{1}{3 !}\left(12 m^{2} \tau^{2}+15 m^{2} \tau^{4}-\frac{14}{3} m^{2} \tau^{6}+\frac{3}{7} m^{2} \tau^{8}\right) \rho^{3}+\mathcal{O}\left(\rho^{4}\right), \\
& c^{ \pm}=1+\left(m \tau^{2}-\frac{1}{6} m \tau^{4}\right) \rho+\frac{1}{2 !}\left(\frac{1}{14} m^{2} \tau^{8}-\frac{8}{9} m^{2} \tau^{6}+3 m^{2} \tau^{4}-2 m^{2} \tau^{2}\right) \rho^{2}+\mathcal{O}\left(\rho^{3}\right), \\
& f=1+\frac{1}{3} m \tau^{4} \rho+\frac{1}{2 !}\left(\frac{1}{7} m^{2} \tau^{8}-\frac{4}{9} m^{2} \tau^{6}+\frac{1}{3} m^{2} \tau^{4}-2 m^{2} \tau^{2}\right) \rho^{2}+\mathcal{O}\left(\rho^{3}\right), \\
& \frac{\xi}{\sqrt{2}}=\left(\frac{1}{2} \tau^{2} m-\frac{1}{4} m \tau^{4}\right) \rho+\frac{1}{2 !}\left(-\frac{1}{28} m^{2} \tau^{8}-\frac{2}{9} \tau^{6} m^{2}+\frac{4}{3} m^{2} \tau^{4}\right) \rho^{2}+\mathcal{O}\left(\rho^{3}\right), \\
& \chi_{2}=\left(4 m \tau^{3}-12 \tau m\right) \rho+\frac{1}{2 !}\left(24 m^{2} \tau-8 m^{2} \tau^{3}+4 \tau^{5} m^{2}-\frac{4}{21} m^{2} \tau^{7}\right) \rho^{2}+\mathcal{O}\left(\rho^{3}\right), \\
& \chi_{h}=\frac{1}{2 !}\left(-\frac{20}{3} \tau^{3} m^{2}+\frac{8}{3} \tau^{5} m^{2}-\frac{20}{63} m^{2} \tau^{7}\right) \rho^{2}+\mathcal{O}\left(\rho^{3}\right) .
\end{aligned}
$$

For completeness the components of the curvature are also given:

$$
\begin{aligned}
& \frac{\Theta_{x}}{\sqrt{2}}=4 m \tau \rho+\frac{1}{2 !}\left(\frac{4}{3} \tau^{5} m^{2}-\frac{8}{3} \tau^{3} m^{2}-12 m^{2} \tau\right) \rho^{2}+\mathcal{O}\left(\rho^{3}\right) \\
& \Theta_{2}=6 m\left(1-\tau^{2}\right) \rho+\frac{1}{2 !}\left(-12 m^{2}+12 m^{2} \tau^{2}-10 \tau^{4} m^{2}+\frac{2}{3} m^{2} \tau^{6}\right) \rho^{2}+\mathcal{O}\left(\rho^{2}\right) \\
& \Theta_{h}=\frac{m^{2}}{2}\left(4 \tau^{2}-\frac{8}{3} \tau^{4}+\frac{4}{9} \tau^{6}\right) \rho^{2}+\mathcal{O}\left(\rho^{3}\right) \\
& \phi=-6 m+\left(-18 m^{2} \tau^{2}+3 \tau^{4} m^{2}\right) \rho+\frac{1}{2 !}\left(-\frac{16}{7} \tau^{8} m^{3}+28 m^{3} \tau^{6}-90 m^{3} \tau^{4}+36 m^{3} \tau^{2}\right) \rho^{2}+\mathcal{O}\left(\rho^{3}\right)
\end{aligned}
$$

Finally, one has that

$$
\begin{aligned}
& 2 \Gamma_{0011}-4 \Gamma_{1010}=2 \xi+\frac{\sqrt{2}}{6} \chi_{2}-2 \sqrt{2} \chi_{h}-\sqrt{2} f, \\
& 2\left(\Gamma_{1100}+\Gamma_{0011}\right)=-\frac{\sqrt{2}}{3} \chi_{2}-2 \sqrt{2} \chi_{h}, \\
& 2 \Gamma_{1100}-4 \Gamma_{0101}=-2 \xi+\frac{\sqrt{2}}{6}-2 \sqrt{2} \chi_{h}+\sqrt{2} f, \\
& 2\left(\Gamma_{0011}-\Gamma_{1100}\right)=3 \xi-\frac{\sqrt{2}}{12} \chi_{2}-\frac{\sqrt{2}}{2} \chi_{h} .
\end{aligned}
$$




\section{Some selected properties of the Jacobi polynomials}

The following properties of the Jacobi polynomials have been used in the calculations described in this article. A good general reference to this matter is the monograph [10].

The Jacobi polynomials are defined by

$$
P_{n}^{(\alpha, \beta)}(\tau)=\frac{\Gamma(\alpha+n+1)}{n ! \Gamma(\alpha+\beta+n+1)} \sum_{m=0}^{n}\left(\begin{array}{c}
n \\
m
\end{array}\right) \frac{\Gamma(\alpha+\beta+n+m+1)}{\Gamma(\alpha+m+1)}\left(\frac{\tau-1}{2}\right)^{m} .
$$

In the above expression the combinatorial coefficients are understood in the generalised sense involving the Gamma function. That is,

$$
\left(\begin{array}{c}
m \\
n
\end{array}\right)=\frac{\Gamma(m+1)}{\Gamma(n+1) \Gamma(m-n+1)}
$$

Derivatives of the above can be rewritten as lower degree Jacobi polynomials via

$$
\frac{\mathrm{d}^{k}}{\mathrm{~d} \tau^{k}} P_{n}^{(\alpha, \beta)}(\tau)=\frac{\Gamma(\alpha+\beta+n+1+k)}{2^{k} \Gamma(\alpha+\beta+n+1)} P_{n-k}^{(\alpha+k, \beta+k)}(\tau) .
$$

The polynomials satisfy the symmetry relation

$$
P_{n}^{(\alpha, \beta)}(-\tau)=(-1)^{n} P_{n}^{(\beta, \alpha)}(\tau) .
$$

The value of the polynomials at $\tau= \pm 1$ is given by

$$
P_{n}^{(\alpha, \beta)}(1)=\left(\begin{array}{c}
n+\alpha \\
n
\end{array}\right), \quad P_{n}^{(\alpha, \beta)}(-1)=(-1)^{n}\left(\begin{array}{c}
n+\beta \\
n
\end{array}\right) .
$$

\section{References}

[1] H. A. Buchdahl, On the compatibility of of relativistic wave equations for particles of higher spin in the presence of a gravitational field, Nuovo Cim. 10, 96-103 (1958).

[2] H. Friedrich, Einstein equations and conformal structure: existence of anti-de Sitter-type space-times, J. Geom. Phys. 17, 125 (1995).

[3] H. Friedrich, Gravitational fields near space-like and null infinity, J. Geom. Phys. 24, 83 (1998).

[4] H. Friedrich, Spin-2 fields on Minkowski space near space-like and null infinity, Class. Quantum Grav. 20, 101 (2003).

[5] H. Friedrich, Smoothness at null infinity and the structure of initial data, in 50 years of the Cauchy problem in general relativity, edited by P. T. Chruściel \& H. Friedrich, Birkhausser, 2004.

[6] A. R. Magid, Differential Galois theory, Notices of the AMS, 1041 (October 1999).

[7] L. J. Mason \& J. P. Nicolas, Conformal scattering and the Goursat problem, J. Hyp. Diff. Eq. 1, 197 (2004).

[8] L. J. Mason \& J. P. Nicolas, Regularity at space-like and null infinity, at gr-qc/0701049, 2007.

[9] P. Sommers, Space spinors, J. Math. Phys. 21, 2567 (1980).

[10] G. Szegö, Orthogonal polynomials, volume 23 of AMS Colloq. Pub., AMS, 1978.

[11] J. A. Valiente Kroon, Polyhomogeneous expansions close to null and spatial infinity, in The Conformal Structure of Spacetimes: Geometry, Numerics, Analysis, edited by J. Frauendiner \& H. Friedrich, Lecture Notes in Physics, page 135, Springer, 2002. 
[12] J. A. Valiente Kroon, Does asymptotic simplicity allow for radiation near spatial infinity?, Comm. Math. Phys. 251 (2004).

[13] J. A. Valiente Kroon, A new class of obstructions to the smoothness of null infinity, Comm. Math. Phys. 244, 133 (2004).

[14] J. A. Valiente Kroon, Time asymmetric spacetimes near null and spatial infinity. I. Expansions of developments of conformally flat data, Class. Quantum Grav. 23, 5457 (2004).

[15] J. A. Valiente Kroon, Time asymmetric spacetimes near null and spatial infinity. II. Expansions of developments of initial data sets with non-smooth conformal metrics, Class. Quantum Grav. 22, 1683 (2005). 